\title{
Plasma-neutral gas simulations of reconnection events in cometary tails
}

\author{
C. Konz ${ }^{1,2,3}$, G. T. Birk ${ }^{1,2}$, and H. Lesch ${ }^{1,2}$ \\ ${ }^{1}$ Institute for Astronomy and Astrophysics, University of Munich, Scheinerstr 1, 81679 Munich, Germany \\ 2 Centre of Interdisciplinary Plasma Science, 85748 Garching, Germany \\ ${ }^{3}$ Max-Planck-Institute for Plasma Physics, Boltzmannstr. 2, 85748 Garching, Germany
}

Received 21 March 2003 / Accepted 17 October 2003

\begin{abstract}
The formation and dynamical evolution of cometary plasma tails and magnetic boundary layers is studied by the first numerical plasma-neutral gas simulations. It is shown that collisional interaction between the cometary neutral envelope and the solar wind plasma leads to the formation of a magnetic barrier that separates the entire cometary body from the solar wind. The overall dynamics, in particular, of the magnetotail are governed by magnetic reconnection. Multiple reconnection events are initiated by the localized excitation of anomalous resistivity modeled by $\eta(j)$. If the comet encounters a heliospheric current sheet, strong disconnection events characterize the cometary plasma tail. But even in the case of homogeneous solar wind conditions, partial disruption of the tail is triggered by dayside reconnection.
\end{abstract}

Key words. comets: general - interplanetary medium

\section{Introduction}

Comets are characterized by the formation of elongated plasma tails. These plasma tails represent magnetic configurations that are very similar to the geomagnetic tail and exhibit a variety of interesting dynamic phenomena (e.g. Mendis \& Ip 1977; Brandt \& Mendis 1978). Among these, probably the most impressive are so-called disconnection events (DE) (Barnard 1899; Niedner \& Brandt 1978; Brandt \& Mendis 1978; Brandt \& Niedner 1987; Cremonese \& Fulle 1988). DEs are characterized by a distinct morphological cyclic sequence. Tail ray generation, tail disconnection and renewal are followed by a relatively quiescent period. Several plasma processes have been proposed to explain the onset of such DEs (Brosius et al. 1987 and references therein). The most promising is magnetic reconnection. Dayside reconnection caused by magnetic field reversals due to crossings of the heliospheric current sheet generally trigger disconnection events (Niedner \& Brandt 1978; Niedner et al. 1981; Niedner \& Schwingenschuh 1987; Verigin et al. 1987; Kirsch et al. 1989; Brandt et al. 1999; Voelzke $\&$ Matsuura 2000). Moreover, in situ measurements of highenergy particles indicate evidence of magnetic reconnection in the front side of Halley's cometosheath (Verigin et al. 1987; Kirsch et al. 1989).

Yi et al. (1994), Brandt et al. (1999) and Voelzke \& Matsuura (2000) found a clear correlation of the DEs observed for comet Halley in 1985-86 and its passing through interplanetary sector boundaries. For this kind of disconnection process,

Send offprint requests to: C. Konz,

e-mail: konz@usm.uni-muenchen.de
Niedner \& Brandt (1978) and Brosius et al. (1987) propose a delay of $0.5-0.75$ days between the encounter with a heliospheric current sheet and the disconnection of the cometary tail.

Numerical magnetohydrodynamic studies have shown that the response of cometary plasmas to magnetic field variations in the ambient medium, as, e.g., tangential discontinuities and variations in solar wind parameters, is characterized by strong dynamic features (Ogino et al. 1986; Schmidt-Voigt 1989; Rauer et al. 1995; Gombosi et al. 1996; Yi et al. 1996; Wegmann 1995). Gombosi et al. performed sophisticated threedimensional ideal MHD simulations of a comet in the solar wind using an adaptive numerical mesh. They succeeded in reproducing density, temperature, and magnetic field profiles measured for Halley's comet by the Giotto probe. Ogino et al. (1986), Schmidt-Voigt (1989), Rauer et al. (1995), and Yi et al. (1996) simulated two- and three-dimensional cases where a comet crosses an interplanetary sector boundary. While Schmidt-Voigt and Rauer et al. did not find indications for magnetic reconnection, Ogino et al. and Yi et al. found clear evidence for magnetic reconnection. Schmidt-Voigt and Rauer et al. performed ideal magnetohydrodynamics (MHD) simulations. In principle, ideal MHD does not allow for field line reconnection, which explains the absence of magnetic reconnection in their simulations. We note, however, that depending on the chosen algorithm, some numerical diffusion can cause uncontrolled magnetic reconnection.

Furthermore, Ip (1985) and Russell et al. (1986) proposed a model with a tailward (nightside) reconnection of magnetic field lines leading to a disconnection of the plasma tail. In such a model, plasmoids are formed in the tail and finally 
expelled by the magnetic slingshot mechanism. Reconnection takes place in the tail current sheet because magnetic field lines are anti-parallel on opposite sides of the tail.

A necessary condition for magnetic reconnection is a local violation of the ideal Ohm's law. As in many astrophysical plasmas, the classical Spitzer resistivity due to Coulomb collisions is of minor importance in the context of reconnection in comets. Rather, anomalous resistivity due to microturbulence triggers the onset of reconnection events (Niedner et al. 1981). In this case momentum transfer between the charged particles is caused by microturbulent electromagnetic fields. Of course, the excitation of localized microturbulent dissipation, usually with some functional dependence on the local current density, may result from processes in the ambient medium, as, e.g., pressure and flow enhancements or magnetic field variations in the solar wind, as, e.g., caused by crossings of an interplanetary sector boundary.

In this paper, we present the first plasma-neutral gas simulations of the formation of cometary tails and boundary layers as well as disconnection phenomena caused by magnetic reconnection using a current-dependent resistivity. Such a localized resistivity is a necessary condition for a realistic modeling of fast, e.g., Petschek-like, reconnection.

Both dayside and nightside reconnection events are investigated. Dayside reconnection is expected to be responsible for DEs associated with crossings of the heliospheric current sheets that can occur up to high latitudes of about $78^{\circ}$ (Brandt \& Snow 2000; Smith et al. 2001). Nightside reconnection, however, can occur independently of changes in the solar wind conditions.

In the next section, the numerical method is introduced. In Sect. 3, the numerical results are shown for the case of a homogeneous solar wind streaming around the comet. In Sect. 4, an encounter of the comet with a heliospheric current sheet is simulated after a well-developed magnetotail has been formed. We discuss our findings in the concluding section.

\section{Numerical model}

We deal with a partially ionized plasma in the fluid description. The ionized fluid component is treated in the framework of magnetohydrodynamics with the addition of collisional momentum transfer with the neutral gas component and thermalization. The fluid equations can be derived from velocity moments of the kinetic Boltzmann equations for the different species (electrons, ions, neutrals). For both fluid components mass balance, momentum balance, and pressure balance equations have to be solved. Our simulations are performed with a 2D version of an explicit finite differences plasma-neutral gas code (Birk \& Otto 1996). The following set of normalized plasma-neutral gas balance equations are integrated numerically together with the induction equation that determines the temporal evolution of the magnetic field:

$$
\begin{aligned}
& \frac{\partial \rho}{\partial t}=-\nabla \cdot(\rho \boldsymbol{v}) \\
& \frac{\partial \rho_{\mathrm{n}}}{\partial t}=-\nabla \cdot\left(\rho_{\mathrm{n}} \boldsymbol{v}_{\mathrm{n}}\right)
\end{aligned}
$$

$$
\begin{aligned}
& \frac{\partial}{\partial t}(\rho \boldsymbol{v})=-\nabla \cdot(\rho \boldsymbol{v} \boldsymbol{v})-\frac{1}{2} \nabla p+(\nabla \times \boldsymbol{B}) \times \boldsymbol{B}-\rho v_{\mathrm{pn}}\left(\boldsymbol{v}-\boldsymbol{v}_{\mathrm{n}}\right) \\
& \frac{\partial}{\partial t}\left(\rho_{\mathrm{n}} \boldsymbol{v}_{\mathrm{n}}\right)=-\nabla \cdot\left(\rho_{\mathrm{n}} \boldsymbol{v}_{\mathrm{n}} \boldsymbol{v}_{\mathrm{n}}\right)-\frac{1}{2} \nabla p_{\mathrm{n}}-\rho_{\mathrm{n}} v_{\mathrm{np}}\left(\boldsymbol{v}_{\mathrm{n}}-\boldsymbol{v}\right) \\
& \frac{\partial p}{\partial t}=-\boldsymbol{v} \cdot \nabla p-\gamma p \nabla \cdot \boldsymbol{v}+(\gamma-1)\left[2 \eta(\nabla \times \boldsymbol{B})^{2}\right. \\
& \left.\quad-3 v_{\mathrm{pn}}\left(p-\frac{\rho}{\rho_{\mathrm{n}}} p_{\mathrm{n}}\right)\right] \\
& \frac{\partial p_{\mathrm{n}}}{\partial t}=-\boldsymbol{v}_{\mathrm{n}} \cdot \nabla p_{\mathrm{n}}-\gamma_{\mathrm{n}} p_{\mathrm{n}} \nabla \cdot \boldsymbol{v}_{\mathrm{n}}-3\left(\gamma_{\mathrm{n}}-1\right) v_{\mathrm{np}}\left(p_{\mathrm{n}}-\frac{\rho_{\mathrm{n}}}{\rho} p\right) \\
& \frac{\partial \boldsymbol{B}}{\partial t}=\nabla \times(\boldsymbol{v} \times \boldsymbol{B})-\nabla \times(\eta \nabla \times \boldsymbol{B}) .
\end{aligned}
$$

In these equations, $\rho, \boldsymbol{v}$, and $p$ are the mass density, fluid bulk velocity, and the thermal pressure. The index $n$ denotes neutral gas quantities. The effective plasma-neutral gas collision frequencies $v_{\mathrm{pn}}$ and $v_{\mathrm{np}}$ fulfill the constraint of momentum conservation, i.e. $v_{\mathrm{pn}} \rho=v_{\mathrm{np}} \rho_{\mathrm{n}}$ with

$$
v_{\mathrm{pn}}=\frac{m_{\mathrm{i}} v_{\mathrm{in}}+m_{\mathrm{e}} v_{\mathrm{en}}}{m_{\mathrm{e}}+m_{\mathrm{i}}}
$$

where $v_{\mathrm{in}}, v_{\mathrm{en}}, m_{\mathrm{e}}$, and $m_{\mathrm{i}}$ are the elastic ion-neutral and electron-neutral collision frequencies as well as electron and ion masses. By $\eta=c^{2} m_{\mathrm{e}} v_{\mathrm{ei}} / 4 \pi v_{\mathrm{A}} L n_{\mathrm{e}} e^{2}$ and $\gamma$ the normalized resistivity, i.e. the normalized inverse magnetic Reynolds number, and the ratio of specific heats are denoted. The equations are normalized by a typical length scale $L$, the Alfvén velocity $v_{\mathrm{A}}=B_{0} / \sqrt{4 \pi \rho_{0}}$ with a typical magnetic field strength $B_{0}$, and the mass density of the ionized fluid component $\rho_{0}$. Other quantities, e.g., the typical (Alfvén) time scale $t_{\mathrm{A}}=L / v_{\mathrm{A}}$, follow in a generic way. The factor $1 / 2$ in front of the pressure force terms in the momentum equations is a consequence of our choice or normalization of the thermal pressures to the magnetic pressure. The Lorentz force in Eq. (3) has been formulated by means of Ampère's law.

For simplicity, both the Hall term as well as the gradient pressure term are neglected in the induction equation.

The physical parameters used in the following simulation runs are: typical length scale $L=5 \times 10^{10} \mathrm{~cm}$, typical plasma particle density $n_{0}=12 \mathrm{~cm}^{-3}$ (mass density $\rho_{0}=m_{\mathrm{i}} n_{\mathrm{o}}$ ), and typical magnetic field strength $B_{0}=5 \times 10^{-3} \mathrm{G}$. The reference values are chosen in a way that the simulated quantities represent a comet at a solar distance of roughly $1 \mathrm{AU}$ (Niedner et al. 1981; Kivelson \& Russell 1995, Chap. 4.2; Flammer et al. 1997). In this normalization, the Alfvén velocity amounts to $v_{\mathrm{A}}=3.15 \times 10^{8} \mathrm{~cm} \mathrm{~s}^{-1}$ and the Alfvén time to $\tau_{\mathrm{A}}=159 \mathrm{~s}$. The length scale $L$ is chosen as the extent of the ionosphere of a typical comet at a solar distance of $1 \mathrm{AU}$.

The two-dimensional simulation runs presented here simulate a system consisting of a dense, cold, neutral, and slab-like comet in a hot, magnetized ambient plasma flow representing the solar wind impinging on the comet's ionosphere. We restrict ourselves to a 2D analysis, since we need a high grid resolution in order to model the reconnection event in a reliable 
way. The main difference to a 3D configuration is the suppression of field line slippage. The slippage is expected to slow down reconnection rather than to hinder it completely (Yi et al. 1996; Konz et al. 2004)

We neglect mass loading of the solar wind, since it is a kinetic effect that cannot be treated consistently in a fluid description. The main effect of mass loading is a broadening of the bow shock (Flammer et al. 1997). In the case of comets the bow shocks are only weak, i.e. temperatures, densities, and magnetic field directions only slightly differ up- and downstream (Gombosi et al. 1996). We, however, exclude the weak bow shock which forms in front of the comet from the simulation region. We rather focus on the subsonic but superalfvénic velocity regime inward of the bow shock and follow the dynamical evolution of the comet's magnetotail and magnetic boundary layer which forms around the comet's front side. Neutral gas desorption from the surface of the comet is, in principle, allowed, but, due to the low average sublimation speed, does not have a significant influence on the dynamics (see also Flammer et al. 1997).

The cometary core is modeled by a density enhancement of the form $\rho_{\mathrm{n}}(r)=\rho_{\min }+\rho_{n_{0}} / \cosh (r / 2)$ localized around the origin of the coordinate system $(r=0)$ with a background density (for numerical reasons) of $\rho_{\min }=1$ and a peak density of $\rho_{n_{0}}=1.5 \times 10^{4}$. We neglect energy transfer from the plasma to the neutral gas and ionization processes. Instead, we assume an ionization equilibrium throughout the simulation region. The enhancement of the neutral gas density is small compared to "real life" comets but still sufficiently large to make sure that the comet's core itself is not affected by the solar wind during the simulation. The neutral gas is assumed to be initially in pressure equilibrium with minimum temperature $T_{n_{0}}=0.1$ at the center of the comet's core. The background plasma is chosen to have an initially homogeneous density $\rho_{\min }=1$ and a temperature which varies between $T_{n_{0}}$ at the comet's core and $T_{0}=100$ in the ambient plasma, following a $T_{0}-\left(T_{0}-T_{n_{0}}\right) / \cosh (r / 2)$ profile similar to the neutral gas temperature. The magnetized solar wind continuously streams into the numerical domain with a constant velocity $v_{y_{0}}=-0.15$ in the negative $y$-direction. In the current normalization, this corresponds to a solar wind velocity of $v_{\text {sol }} \approx 470 \mathrm{~km} \mathrm{~s}^{-1}$ which is comparable to typical solar wind velocities (cf. Kivelson \& Russell 1995, Chap. 4.2). The initial magnetic field is taken to be homogeneous with $\boldsymbol{B}=B_{x_{0}} \hat{x}$ where $B_{x_{0}}=0.01$ is chosen to yield typical solar wind magnetic fields of $50 \mu \mathrm{G}$. This corresponds to a local Alfvén Mach number of the solar wind of $M_{\mathrm{A}}=15$. Throughout the simulation, plasma velocity and density as well as magnetic field strength are kept constant at the upper $y$-boundary, by this simulating a continuous inflow of magnetized solar wind plasma. The effective plasma-neutral gas collision frequency $v_{\mathrm{pn}}$ is given by the classical temperature and neutral gas density dependent model $v_{\mathrm{pn}}=v_{\mathrm{c}} \rho_{\mathrm{n}} T^{1 / 2}$ (Huba 1998) where the constant prefactor is $v_{\mathrm{c}}=8 \times 10^{-6}$.

The numerical box is given by $x \in[-60,60]$ and $y \in$ $[-250,30]$ in units normalized to $L$. The numerical grid is resolved by $303 \times 703$ grid points. For the boundary conditions, extrapolation is chosen at any but the upper $\left(y_{\max }\right)$ boundary where a constant inflow of the fully ionized fluid component is prescribed. Magnetic reconnection demands for some localized violation of ideal Ohm's law. We model a current dependent resistivity of the form

$$
\eta(j)=\eta_{1}+\eta_{2}\left[j^{2}-j_{\text {crit }}^{2}\right] \Theta\left(j^{2}-j_{\text {crit }}^{2}\right)
$$

with a small constant background resistivity $\eta_{1}=10^{-5}$ (in order to control the influence of numerical resistivity $\eta_{\text {num }}<10^{-6}$ ) and an anomalous, current density dependent resistivity which sets in above some critical current density $j_{\text {crit }}$ (cf. Papadopoulos 1977). In our case, the numerical resistivity is negligible on the time scales considered. The critical current density was heuristically chosen as $j_{\mathrm{c}}=0.1$ while the coefficient is chosen to be $\eta_{2}=0.05$. The formation of localized nonideal regions in dependence on the electric current is motivated by the concept of anomalous resistivity due to microturbulence where momentum transfer between the charged species is caused by turbulent electromagnetic fields (e.g., Huba 1985; Benz 1993).

Starting from the initial configuration, we follow the dynamical evolution of a cometary tail in the solar wind with constant magnetic polarity over some 2500 Alfvén times in the first simulation presented here. The second simulation in this paper starts from a situation of a fully developed magnetotail which is taken from the first simulation after approximately 700 Alfvén times. This latter simulation investigates the dynamical evolution of such a fully developed tail when the comet crosses the heliospheric current sheet.

\section{Numerical results: Run I - Homogeneous solar wind flow}

In this section, we present results from a simulation of the formation of cometary boundary layers and magnetotails caused by a homogeneously streaming solar wind plasma with a constant polarity of the magnetic field. The simulation starts from the aforesaid initial configuration of a dense, cold neutral gas cloud in a hot, magnetized ambient plasma flow representing the solar wind. Figure 1 shows the evolution of the magnetic field, the plasma flow, and the resistivity after $t=2 \tau_{\mathrm{A}}$, $t=300 \tau_{\mathrm{A}}$, and $t=904 \tau_{\mathrm{A}}$ respectively. The associated formation of current sheets is displayed in Fig. 2 for the current density $j_{z}$ after $t=602 \tau_{\mathrm{A}}, t=904 \tau_{\mathrm{A}}$, and $t=1494 \tau_{\mathrm{A}}$ while the corresponding evolution of the magnetic field strength $B$ is presented in Fig. 3 for the time steps $t=300 \tau_{\mathrm{A}}, t=904 \tau_{\mathrm{A}}$, and $t=1201 \tau_{\mathrm{A}}$, respectively. Collisional plasma-neutral gas interaction due to the last term on the rhs of Eq. (3) leads to a stagnation of the solar wind flow at the front side of the comet. Consequently, a strong magnetic boundary layer forms. As long as the current density in the system does not exceed the critical value $j_{\text {crit }}$, magnetic field lines are frozen into the streaming motion of the plasma and therefore compressed in front of the comet and draped around the comet's sides as the plasma streams by it. The compressed field in front of the comet forms a so-called magnetic barrier which is a boundary layer with enhanced magnetic field strength. A magnetotail forms in a well defined way as is expected for non-magnetized objects (planets like Venus, Jovian moons, comets) that are 

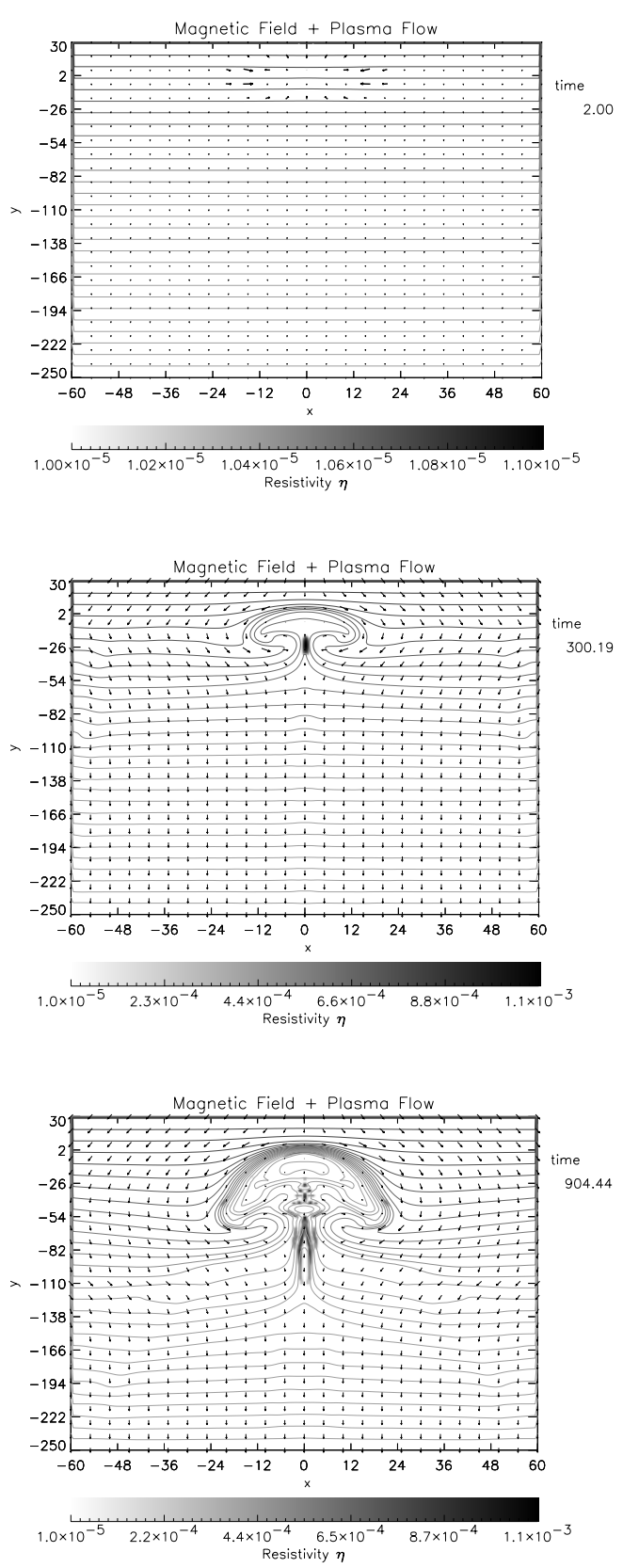

Fig. 1. The magnetic field (dashed lines), the plasma flow (arrows), and the resistivity (grey scales) after $t=2 \tau_{\mathrm{A}}, t=300 \tau_{\mathrm{A}}$, and $t=904 \tau_{\mathrm{A}}$, respectively, for the case of a constant polarity of the magnetic field of the solar wind.

passed by a magnetized plasma flow (cf. Alfvén 1957). The formation of Alfvén wings can be identified in the middle and bottom subfigures of Fig. 3 . Their angle to the main magnetotail slowly decreases with time (compare also Fig. 4, top subfigure). The formation of the magnetic barrier and the magnetotail is connected to the generation of strong currents which eventually exceed the critical current density followed by the onset of an anomalous localized resistivity. Initially, anomalous resistivity shows up in the magnetotail at $t \approx 300 \tau_{\mathrm{A}}$ where anti-parallel field lines form a strong current sheet.

Later on, the current sheet in the magnetic barrier also becomes resistive. Both regions are then subject to magnetic
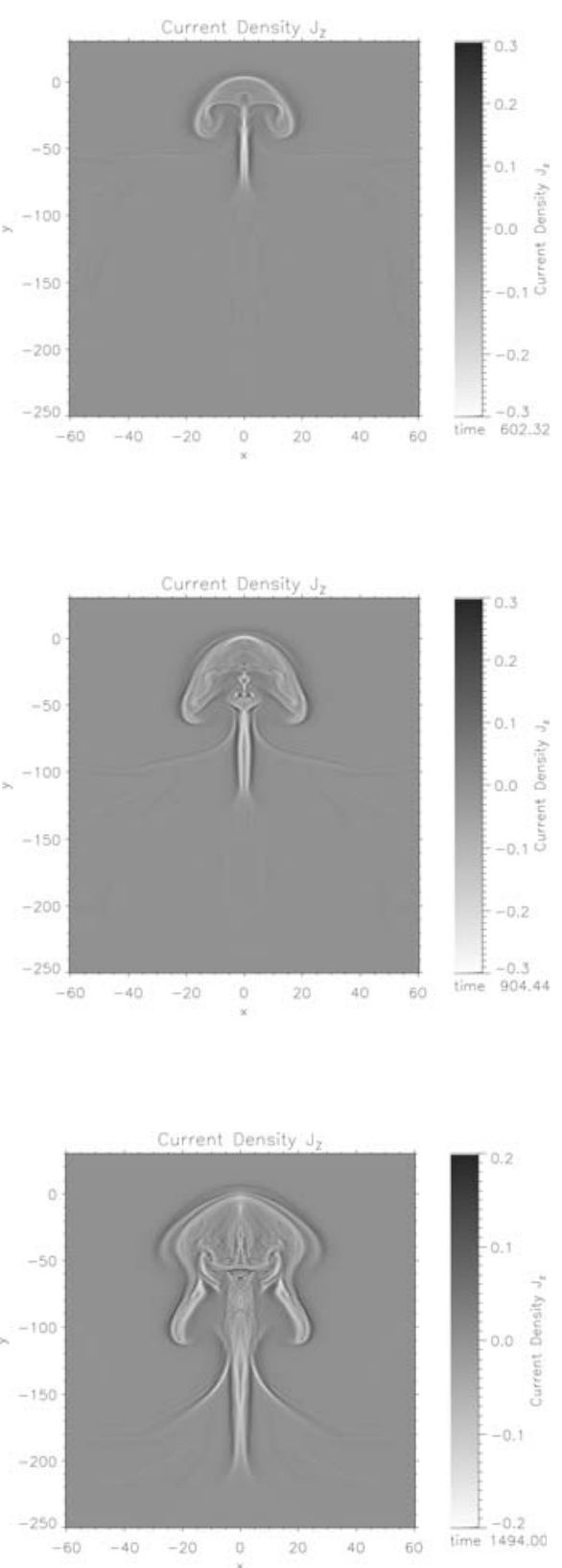

Fig. 2. The current density $j_{z}$ after $t=602 \tau_{\mathrm{A}}, t=904 \tau_{\mathrm{A}}$, and $t=1491 \tau_{\mathrm{A}}$, respectively, for the case of a constant polarity of the magnetic field of the solar wind.

diffusion and reconnection. Nightside reconnection of the magnetic field quickly leads to the formation of an X-point in the cometary tail (see last subfigure of Fig. 1), followed by the disconnection of the far part of the magnetotail. In the magnetic barrier, the anomalous resistivity leads to a diffusion of the magnetic field resulting in a slight broadening of the barrier and a slow diffusion of the field lines through the comet's core. This diffusion initiates the splitting of the magnetic barrier into an inner and an outer layer at $t \approx 1700 \tau_{\mathrm{A}}$ (see first subfigure of Fig. 4).

The broadening of the magnetic barrier due to magnetic diffusion and its splitting into two layers can be seen in Fig. 5 showing the magnetic field strength at $t=1787 \tau_{\mathrm{A}}$ and 

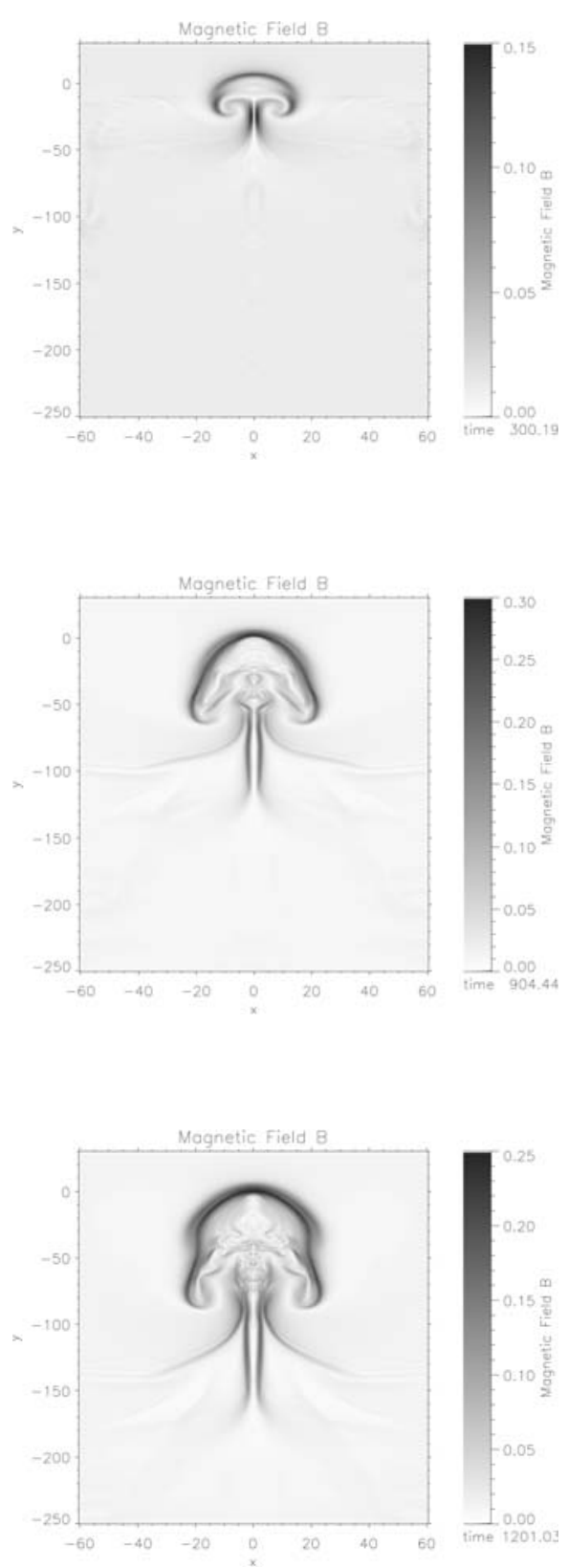

Fig. 3. The magnetic field strength $B$ after $t=300 \tau_{\mathrm{A}}, t=904 \tau_{\mathrm{A}}$, and $t=1201 \tau_{\mathrm{A}}$, respectively, for the case of a constant polarity of the magnetic field of the solar wind.

$t=2080 \tau_{\mathrm{A}}$. In the standard picture of nightside reconnection (e.g., Russell et al. 1986), the far end of the magnetotail is disconnected and expelled by the magnetic slingshot mechanism. The results, presented here, however show that even after the topological disconnection by the formation of an X-point, the tail may stay attached to the comet. The resulting magnetic field structure may account for features like knots which are often observed in plasma tails. In fact, the disconnection event for the case of a constant polarity of the solar wind is a much more complicated process than the intuitive idea of a sling might suggest.

The Figs. 4, 6, and 7 show the magnetic field, its strength, the plasma flow, the resistivity, and the current density for this process of tailward disconnection. Figures 3 and 4 exhibit that
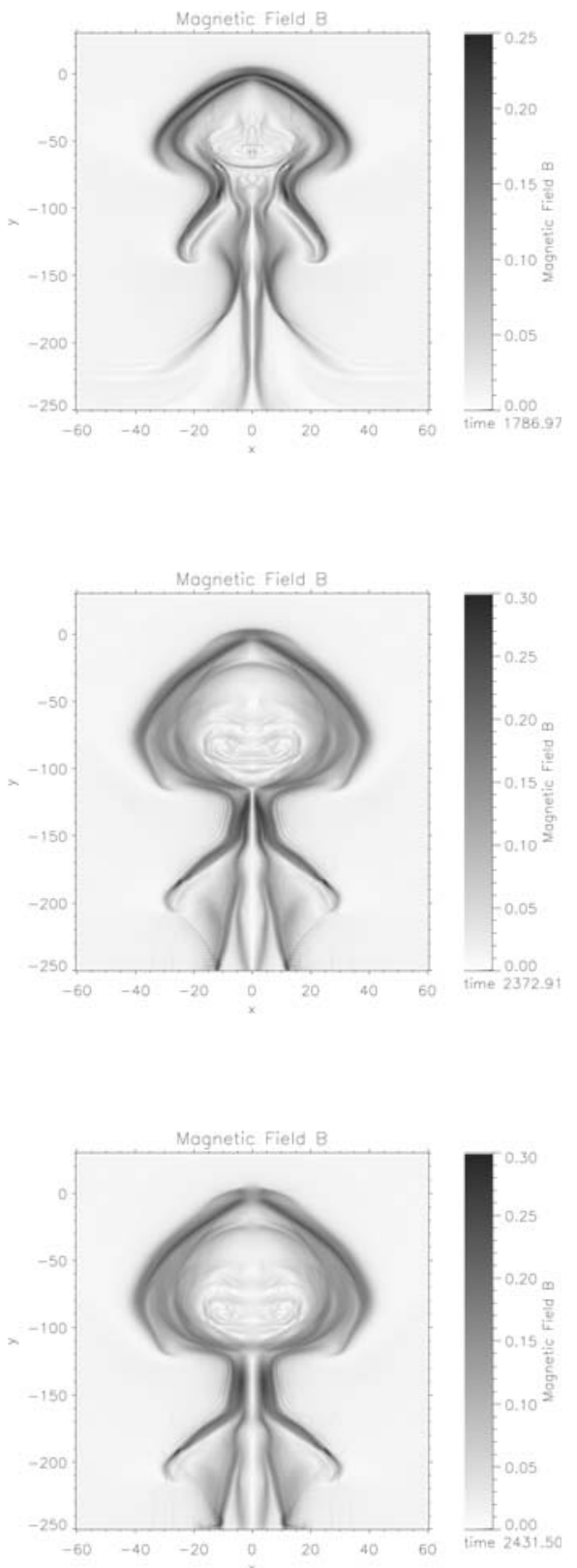

Fig. 4. The magnetic field strength $B$ after $t=1787 \tau_{\mathrm{A}}, t=2373 \tau_{\mathrm{A}}$, and $t=2432 \tau_{\mathrm{A}}$, respectively, for the case of a constant polarity of the magnetic field of the solar wind.

the magnetic boundary layer around the comet is elongated during the simulation due to further accumulation of magnetic flux while the comet moves through the solar wind. The originally very localized magnetic barrier extends at $t \approx 1200 \tau_{\mathrm{A}}$ down to almost $y=-90$, thus forming an outer shield around the part of the magnetotail which is closest to the comet, including the X-point. The specific morphology of this outer magnetic shield with its inward curved wings exerts a stabilizing magnetic pressure on the region around the $\mathrm{X}$-point. Therefore, the tail stays attached to the comet although the X-point slowly moves downstream (Figs. 1 and 6). The elongation of the outer barrier continues until it finally becomes unstable to pinching (last subfigure in Fig. 3 and first one in Fig. 4). As in the Sweet-Parker model for 2D reconnection, plasma streams 

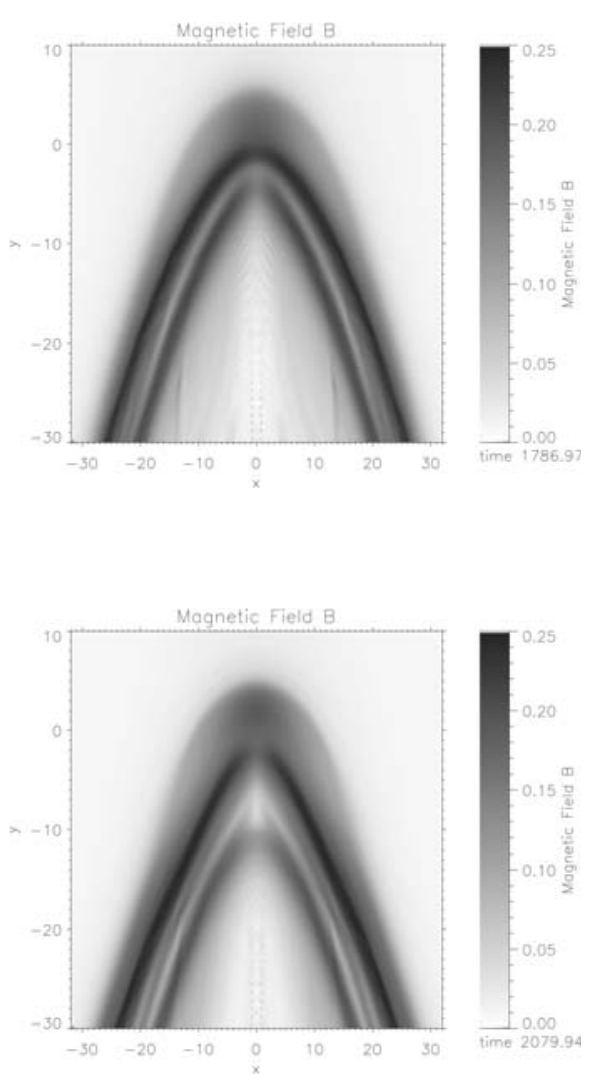

Fig. 5. The magnetic field strength $B$ in the diffusion region after $t=1787 \tau_{\mathrm{A}}$ and $t=2080 \tau_{\mathrm{A}}$ for the case of a constant polarity of the magnetic field of the solar wind.

into the X-point from the sides carrying magnetic flux with it and thus pinching the outer magnetic barrier. The pinching proceeds down to the original width of the inner tail (middle subfigures in Figs. 4 and 6), thereby creating a strong current sheet (Fig. 7) at $t \approx 2373 \tau_{\mathrm{A}}$ which finally dominates the tail dynamics. The high current density is related to a finite anomalous resistivity and the onset of Petschek-type magnetic reconnection (last two subfigures in Fig. 8). At the end of the simulation, the strong current sheet in the pinched region has been disrupted by magnetic reconnection. No outer magnetic shield can any longer prevent the disconnection of the far part of the magnetotail which is now transported downstream at the velocity of the solar wind. The onset of Petschek-type reconnection in the pinched region signals the final disconnection event. It takes about 1000 Alfvén times from the initial phase of the pinching of the outer layer to the disconnection of the tail. This corresponds to a period of about 1.8 days considering the used normalization time scale, a period which is slightly longer than the disconnection time scales observed with crossings of the heliospheric current sheet.

Apart from the nightside reconnection, dayside magnetic diffusion also plays an important role in the dynamics of cometary tails. Anomalous resistivity in the magnetic barrier due to microturbulence leads to a diffusion of magnetic flux through the comet's ionosphere followed by the aforementioned splitting of the magnetic barrier. By this, the inner layer slowly loses its connection to the cometary ionosphere and
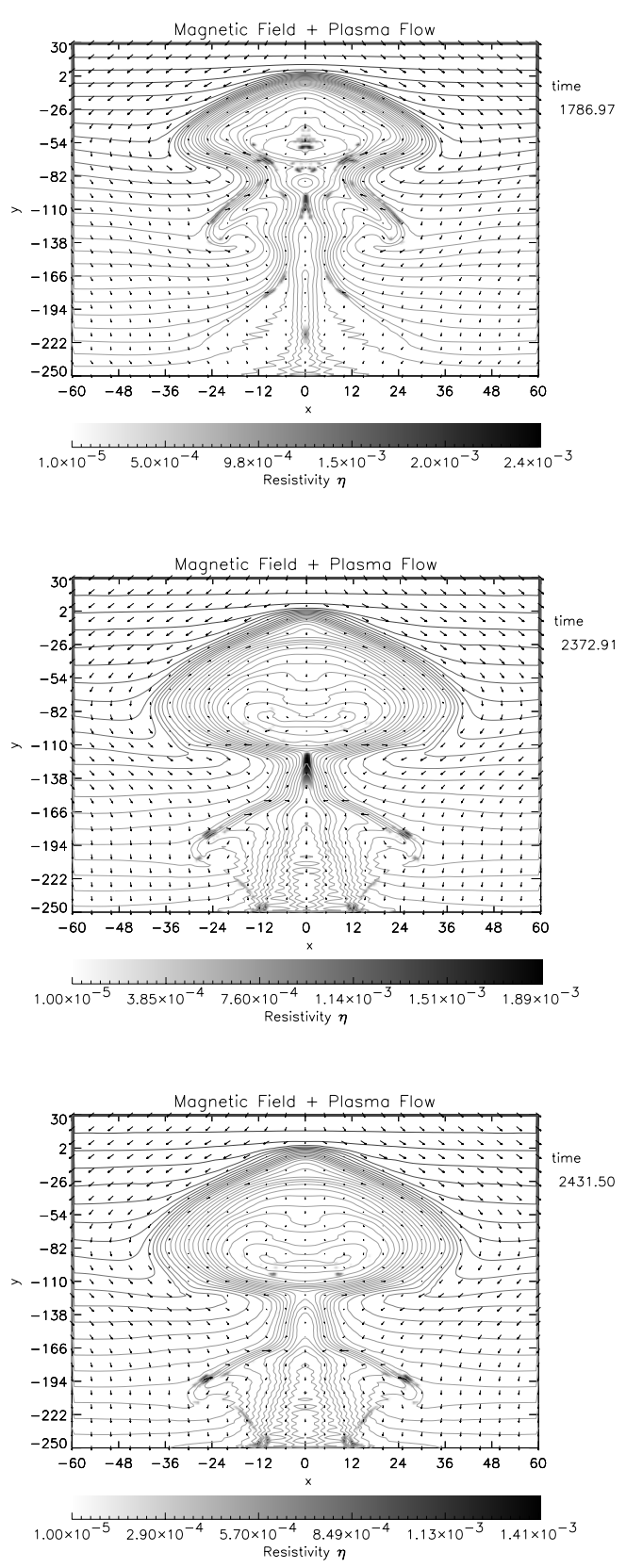

Fig. 6. The magnetic field (dashed lines), the plasma flow (arrows), and the resistivity (grey scales) after $t=1787 \tau_{\mathrm{A}}, t=2373 \tau_{\mathrm{A}}$, and $t=2432 \tau_{\mathrm{A}}$, respectively, for the case of a constant polarity of the magnetic field of the solar wind.

falls back behind the comet. Figures 5 and 9 show this process where the inner barrier diffuses into the region behind the comet, broadens, and finally disappears. A similar effect can be observed for the outer layer at a later time of the simulation (Fig. 9). This diffusion weakens the connection of the magnetotail to the comet which can be noted by the creation of a magnetic cavity (bright central region in Figs. 5 and 9) between the inner and outer barrier. This cavity grows quickly and finally leads to a disconnection of the inner layer from the comet. The cometary tail becomes too "heavy" to stay completely connected to the comet. 

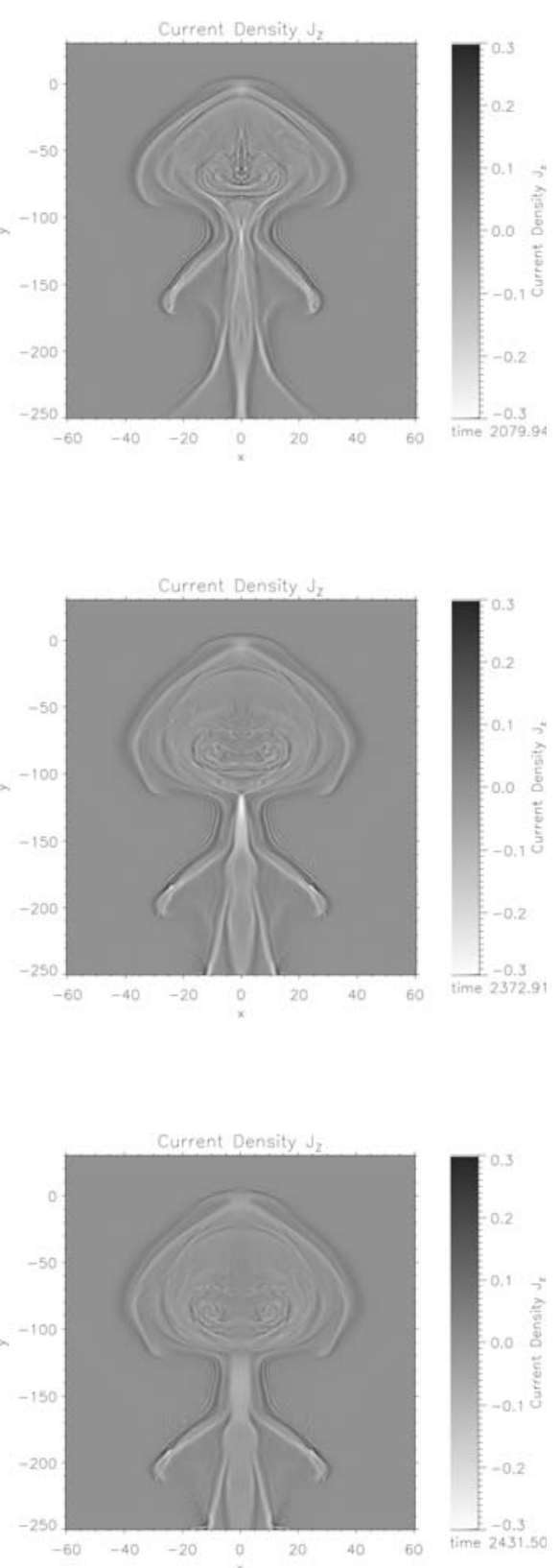

Fig. 7. The current density $j_{z}$ after $t=2080 \tau_{\mathrm{A}}, t=2373 \tau_{\mathrm{A}}$, and $t=2432 \tau_{\mathrm{A}}$, respectively, for the case of a constant polarity of the magnetic field of the solar wind.

Summarizing, we find two mechanisms for disconnection events in a solar wind with constant wind properties and field polarity: nightside reconnection and dayside diffusion of the magnetic field. Both lead to a disconnection of the inner part of the magnetotail whose magnetic flux has been accumulated at the early stages of the simulation. The more recently accumulated flux stays connected to the comet's ionosphere and will form a new magnetic barrier and tail which will then again be subject to magnetic diffusion and reconnection. The overall process of disconnection events by this becomes quasicyclic. Since we did not include mass-loading of the solar wind and outgasing from the comet's surface, no plasma density enhancement can be seen in the ejected tail. However, increased Ohmic dissipation $\eta j^{2}$ at the pinched region can account for a
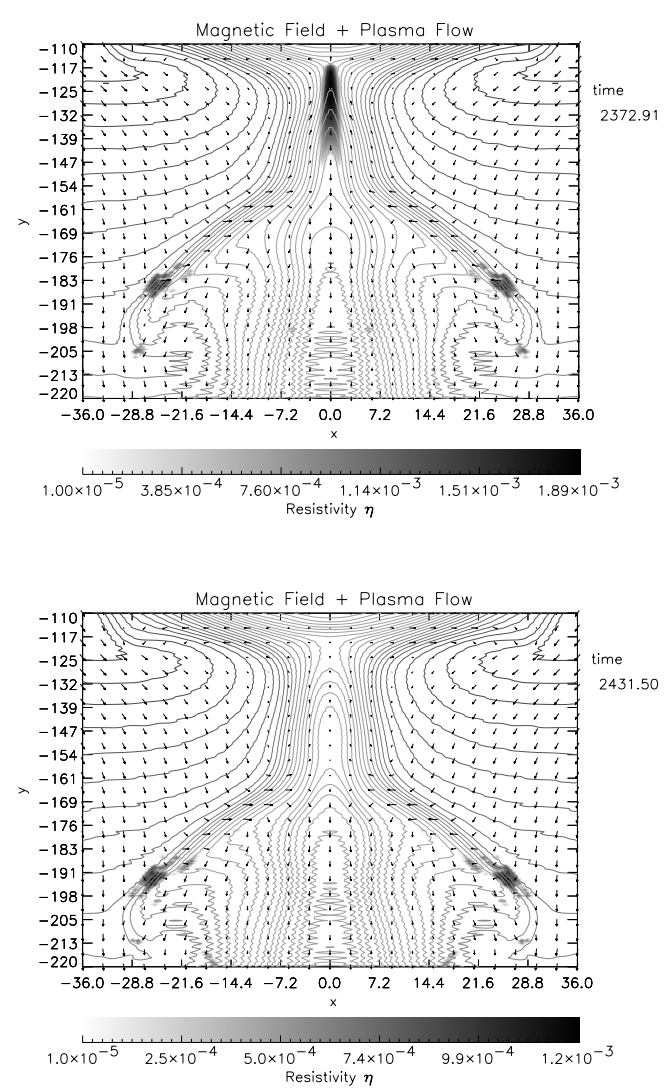

Fig. 8. The magnetic field (dashed lines), the plasma flow (arrows), and the resistivity (grey scales) in the disconnection region after $t=2373 \tau_{\mathrm{A}}$ and $t=2432 \tau_{\mathrm{A}}$ for the case of a constant polarity of the magnetic field of the solar wind.

brightening of the disconnected tail head. Including ionization processes and outgasing of neutrals is necessary to end up with a plasmoid like density enhancement of the tail plasma. Finally, Fig. 10 shows that the neutral cometary core largely remains unaffected by the impact of the solar wind plasma. This is due to the high density contrast between the cometary core and the solar wind plasma.

\section{Numerical results: Run II - Encounter with a heliospheric current sheet}

In this section, we discuss the dynamics of a well-established cometary tail and magnetic barrier after an encounter with a heliospheric current sheet. We start from a configuration of the previous simulation where both magnetotail and magnetic barrier are well developed and nightside reconnection has not yet fully set in $\left(t \approx 705 \tau_{\mathrm{A}}\right)$. The $x$-component of the magnetic field is then multiplied by a factor $-\tanh \left(y-y_{0}\right) / 1.5$, representing an interplanetary sector boundary at $y=y_{0}$ which in the presented simulation is chosen to be 25 . The current sheet is shown in the first subfigure of Fig. 11. For better viewing, the current sheet only has been plotted with a ten times enhanced current density. Different to previous authors (e.g., Rauer et al. 1995; Schmidt-Voigt 1989) we assume a continuous variation of the magnetic field in the current sheet as in a Harris sheet. Considering the spatial extent of the heliospheric current sheet, 

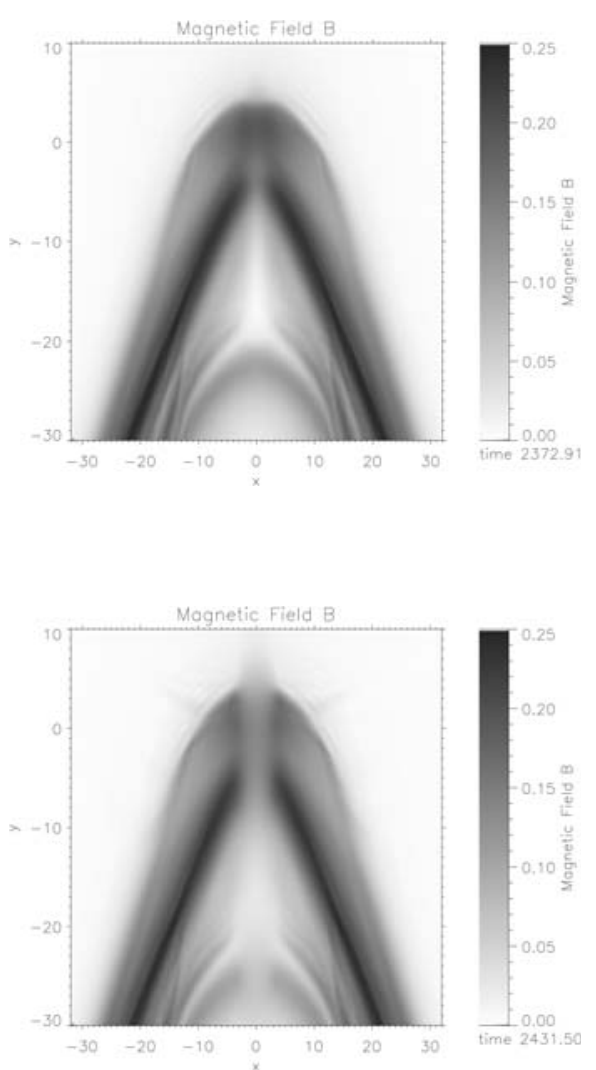

Fig. 9. The magnetic field strength $B$ in the diffusion region after $t=2373 \tau_{\mathrm{A}}$ and $t=2432 \tau_{\mathrm{A}}$ for the case of a constant polarity of the magnetic field of the solar wind.

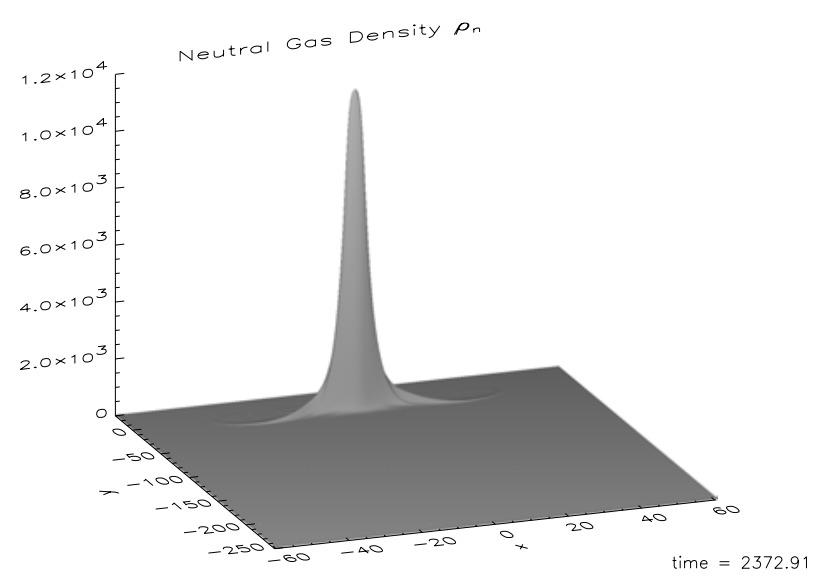

Fig. 10. The neutral gas blob representing the cometary core after $t=2373 \tau_{\mathrm{A}}$.

such a modeling seems to be more realistic than a tangential discontinuity model. The magnetic field at the upper boundary is also reversed: $\boldsymbol{B}=-B_{x_{0}} \hat{x}$ while all other parameters, including the inflow velocity $v_{y_{0}}$ are the same as in the first simulation.

Figures 12 and 13 show the magnetic field, the plasma flow, and the resistivity at several timesteps after the encounter of the comet with the heliospheric current sheet. The gap in the sequence of field lines indicates the position of the sector boundary. Figure 12 demonstrates how the current sheet approaches the comet and finally passes it. In its wake, magnetic flux with reversed polarity is carried along with the solar
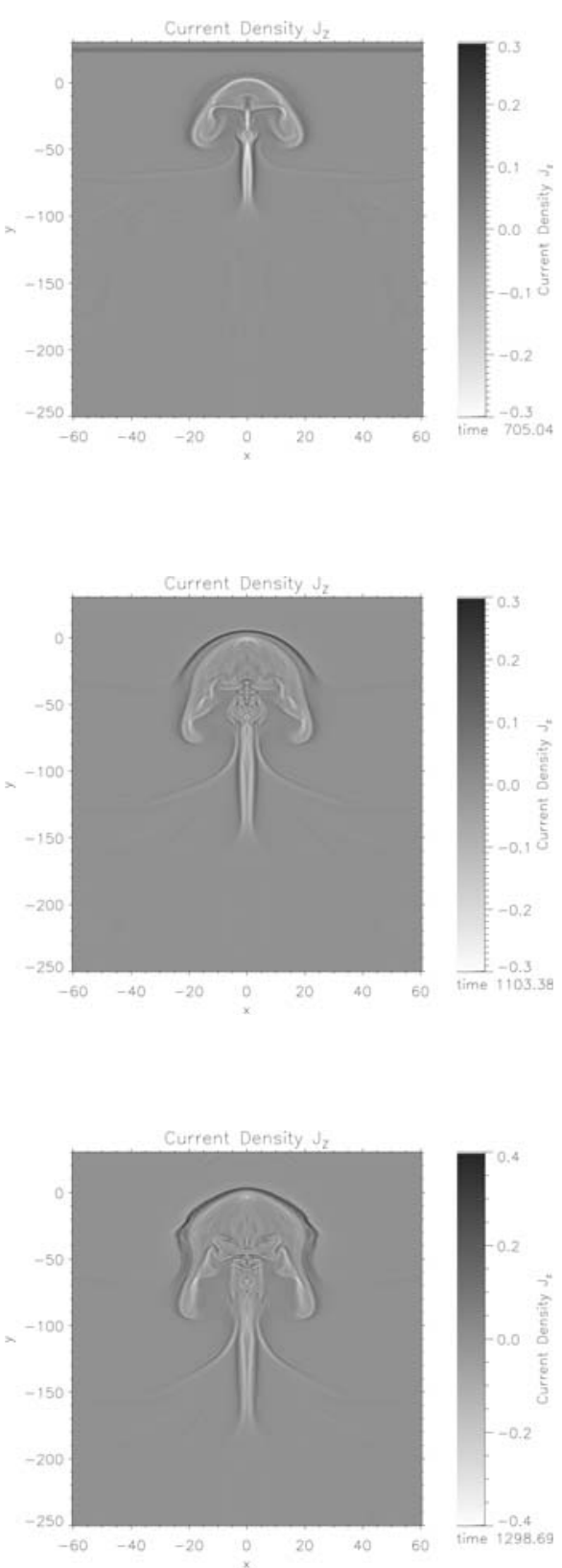

Fig. 11. The current density $j_{z}$ after $t=705 \tau_{\mathrm{A}}, t=1103 \tau_{\mathrm{A}}$, and $t=1299 \tau_{\mathrm{A}}$, respectively, for the case of a reversal of the magnetic field of the solar wind.

wind and is compressed in front of the existing magnetic barrier, thereby creating a second boundary layer with a reversed field (cf. Fig. 14) and a strong current sheet (dark gray) between the two boundary layers (see Fig. 11). The current density in this current sheet largely exceeds the critical current density, thus yielding a strong anomalous resistivity (cf. middle and bottom subfigures in Fig. 12). Such a situation with magnetic field reversal across a resistive current sheet is subject to rapid magnetic reconnection. Reconnection of magnetic field lines sets in at about $t=1300 \tau_{\mathrm{A}}$ (bottom subfigure in Fig. 12). However, the field lines are not reconnected across the symmetry axis of the system but rather at the lateral edges of the original magnetic barrier at $x \approx \pm 20$ where the gradient of the resistivity along the field lines is largest (cf. Eq. (7)). We stress 

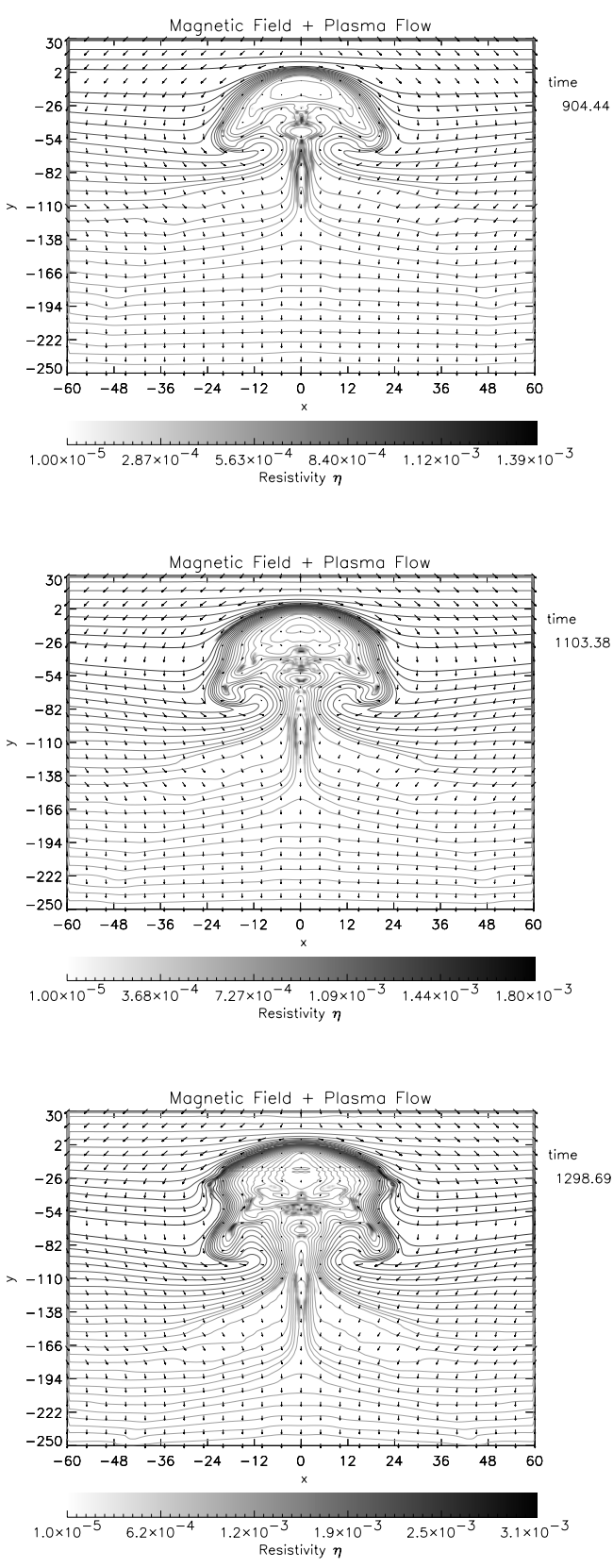

Fig. 12. The magnetic field, the plasma flow, and the resistivity after $t=904 \tau_{\mathrm{A}}, t=1103 \tau_{\mathrm{A}}$, and $t=1299 \tau_{\mathrm{A}}$, respectively, for the case of a reversal of the magnetic field of the solar wind.

that for steep gradients of resistivity that occur during the dynamical evolution the $\nabla \eta \times(\nabla \times \boldsymbol{B})$ contribution to the final term of the induction Eq. (7) can dominate for the magnetic reconnection. Thus, magnetic flux is peeled off like onion skins from the sides of the magnetic barrier (upper two subfigures in Figs. 13 and 16) resulting in a violent disconnection of parts of the magnetotail. Simultaneously, the original X-point in the tail collapses to an extended strong current sheet like in the previous simulation (middle subfigures in Figs. 13, 15, and 16) initiating rapid magnetic reconnection in the central region of the tail. But apart from this, the magnetic reconnection of the sides of the magnetic barrier dominates the dynamics of the tail. After the reconnection of the upper part of the magnetic
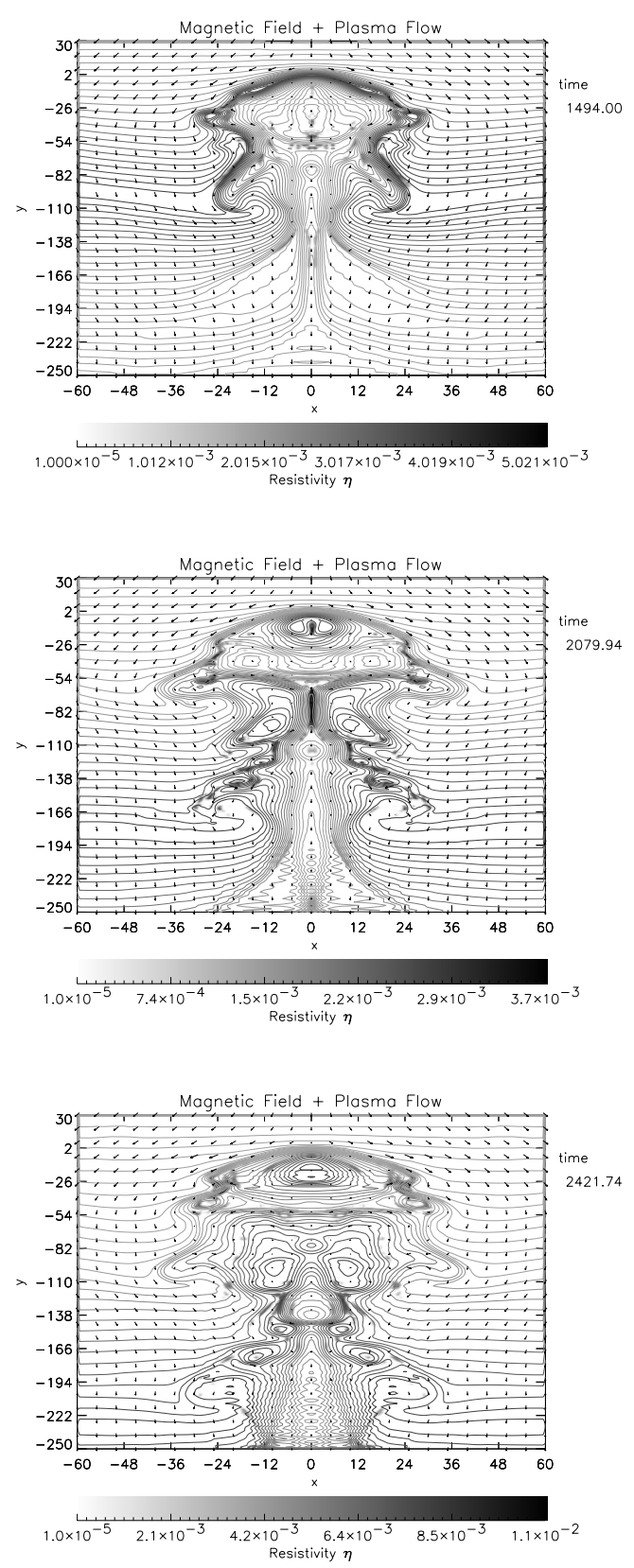

Fig. 13. The magnetic field, the plasma flow, and the resistivity after $t=1494 \tau_{\mathrm{A}}, t=2080 \tau_{\mathrm{A}}$, and $t=2422 \tau_{\mathrm{A}}$, respectively, for the case of a reversal of the magnetic field of the solar wind.

barrier the lower part also becomes subject to magnetic reconnection. Finally, the entire magnetic barrier along the comet's sides is destroyed by magnetic reconnection and dissipated. At the end of the simulation (last subfigure in Fig. 13) the magnetotail has been topologically completely disconnected from the comet. Only comparably weak magnetic fields and current densities remain in large parts of the region of the former magnetic barrier (last subfigures in Figs. 15 and 16). However, the build-up of a new magnetic barrier, with reversed polarity, can be conceived at the end of the simulation (cf. Fig. 16).

Roughly 400 Alfvén times pass from the first contact of the current sheet with the comet's magnetic barrier and the onset of magnetic reconnection. In the chosen normalization, this 

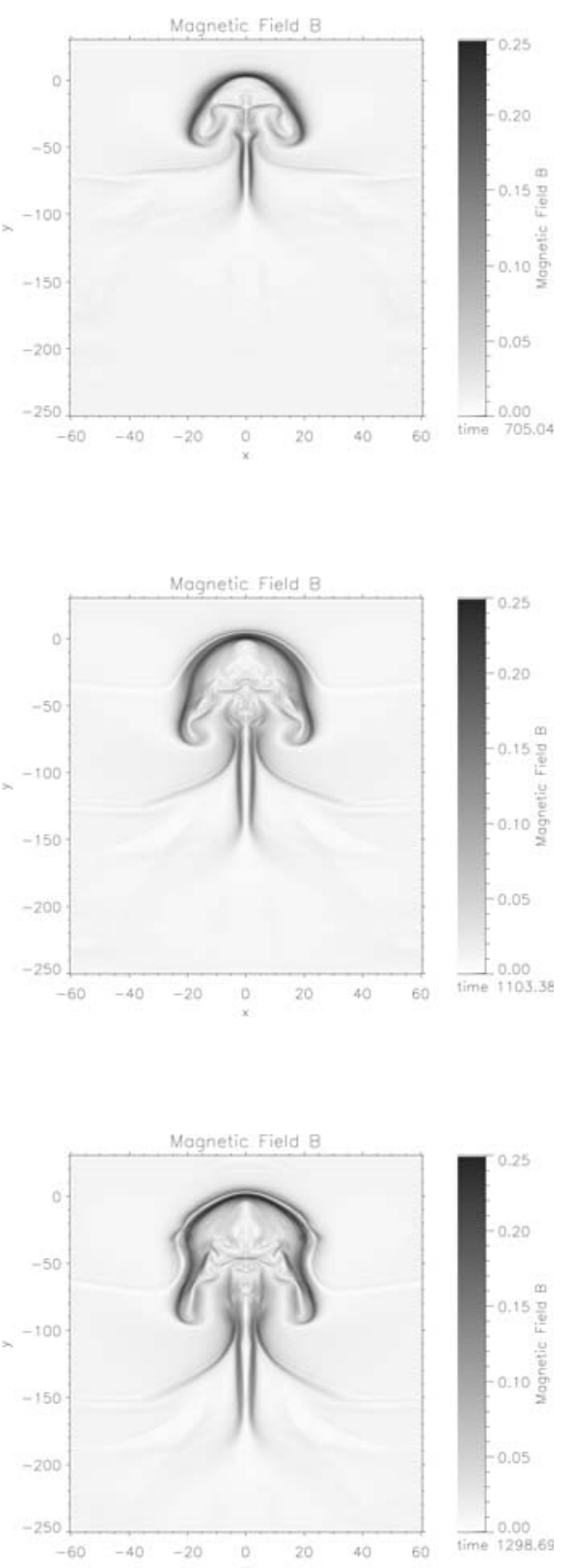

Fig. 14. The magnetic field strength $B$ after $t=705 \tau_{\mathrm{A}}, t=1103 \tau_{\mathrm{A}}$, and $t=1299 \tau_{\mathrm{A}}$, respectively, for the case of a reversal of the magnetic field of the solar wind.

corresponds to approximately 18 hours, i.e. the period suggested by Niedner \& Brandt (1978) and Brosius et al. (1987). Our findings corroborate the results of 3D pure MHD simulations that indicate that the HCS crossings can cause DEs (Yi et al. 1996). Measurements by the Vega spacecraft have shown that in the case of Halley, DEs can be linked to HCS crossings and the magnetic field polarity in the magnetic barrier reverses between different HCS encounters, as is expected within the dayside reconnection scenario (Niedner \& Schwingenschuh 1987). In addition, the observed acceleration of charged particles in the vicinity of Halley (Verigin et al. 1987; Kirsch et al. 1989) supports the reconnection model. We cannot agree with the view proposed by Wegmann (1995) that it may be possible to explain DEs on the grounds of ideal MHD processes.
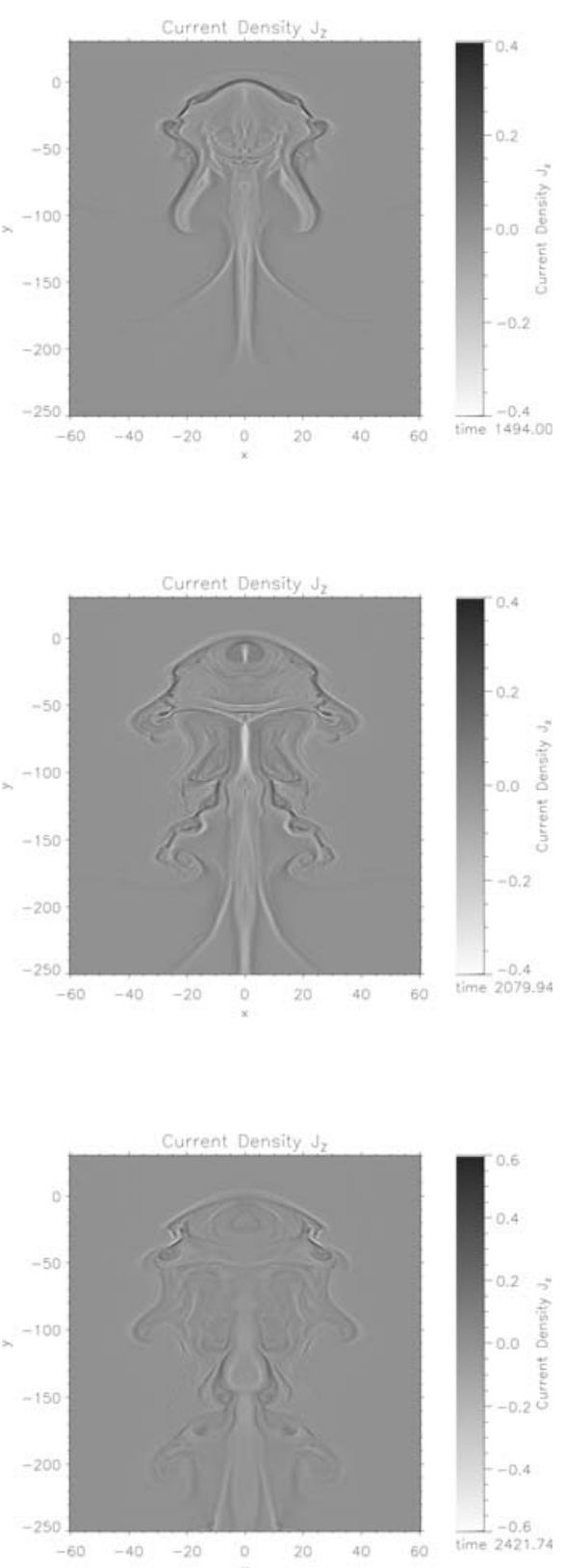

Fig. 15. The current density $j_{z}$ after $t=1494 \tau_{\mathrm{A}}, t=2080 \tau_{\mathrm{A}}$, and $t=2422 \tau_{\mathrm{A}}$, respectively, for the case of a reversal of the magnetic field of the solar wind.

The main difference between this dayside reconnection and the nightside reconnection of the first simulation lies in the fact that magnetic reconnection at the comet's nightside always leads to central disconnection of the comet's magnetotail. Dayside reconnection leads to DEs with parts of the magnetotail being peeled off from the sides. Furthermore, dayside reconnection is more violent, yielding about 2.5 times higher resistivities and by this also higher reconnection rates than in the nightside case. Therefore, disconnection time scales for dayside reconnection are shorter than those for nightside reconnection. Without doubt, the strongest disconnection events are likely connected with passings of interplanetary sector boundaries as suggested by Niedner \& Brandt (1978). 

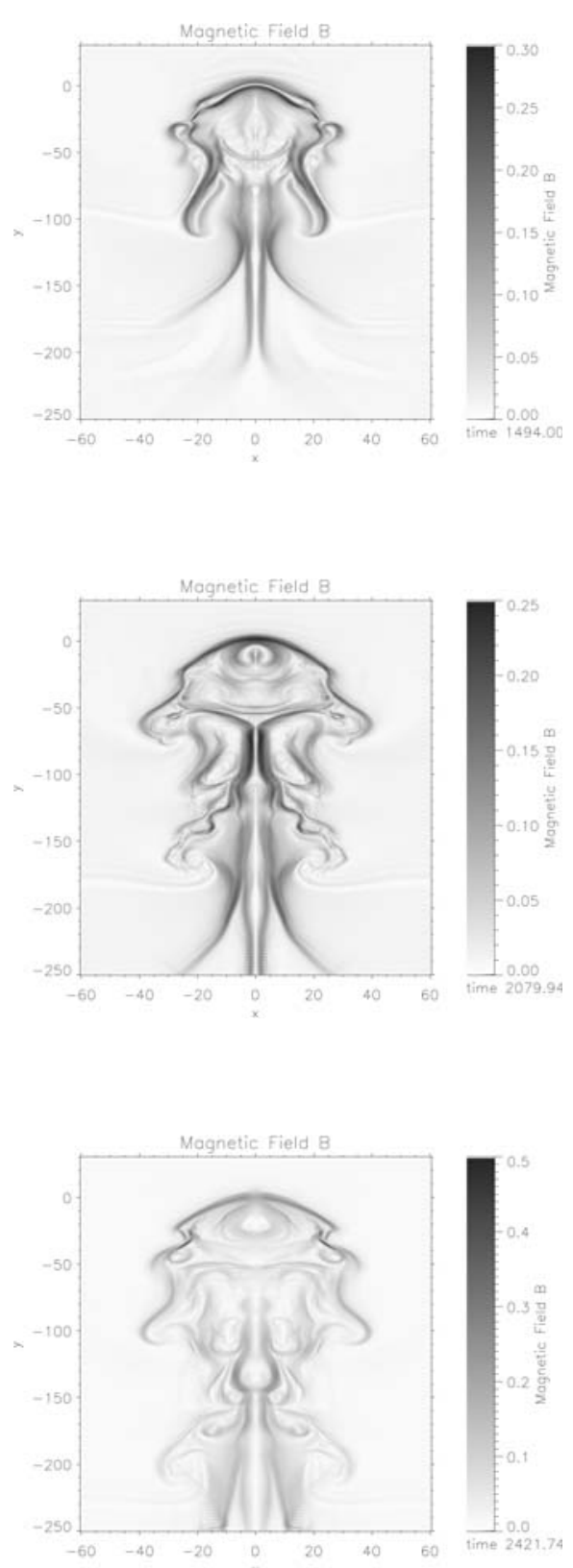

Fig. 16. The magnetic field strength $B$ after $t=1494 \tau_{\mathrm{A}}, t=2080 \tau_{\mathrm{A}}$, and $t=2422 \tau_{\mathrm{A}}$, respectively, for the case of a reversal of the magnetic field of the solar wind.

In our simulations the chosen symmetry of the solar wind with respect to the cometary tail results in symmetric DEs. In the case of a change of the solar wind direction or an asymmetric solar wind inflow the dayside reconnection is expected to produce asymmetric DEs with tail rays on one side only (see examples for DEs in Brandt et al. 1999).

\section{Conclusions}

The interaction of the hot solar wind plasma with an unmagnetized body, be it a planet like Venus or a comet like Giacobini-Zinner, results in the formation of magnetic barriers and magnetic tails. We presented the first plasma-neutral gas simulations for the formation of both the cometopause (collisionopause) that separates the hot solar wind plasma from the weakly ionized cometary gas and the cometary plasmatail characterized by a draped magnetic field and associated current sheets. The magnetic barrier plays an important role in the dynamical stabilization of the comets since it leads to a partial deflection of the solar wind. In the magnetic barrier, microturbulence leads to an anomalous resistivity. For homogeneous solar wind conditions without magnetic field reversals, magnetic diffusion results in a slight broadening of the boundary layer. More important, this diffusion initiates the differentiation of the magnetic barrier into an inner and an outer layer, by this partly cutting the tail's connection to the comet.

As the comet encounters a heliospheric current sheet, magnetic flux with reversed polarity is carried along with the solar wind and compressed in front of the existing magnetic barrier. Consequently, a second boundary characterized by a strong current sheet forms. Measurements by the Vega probes have shown that such magnetic field configurations form in the case of the Halley comet (Niedner \& Schwingenschuh 1987).

This configuration is subject to rapid magnetic reconnection with the field lines being reconnected at the lateral edges of the original magnetic barrier. Magnetic flux is disconnected from the sides of the magnetic barrier which results in a violent partial disconnection of the cometotail. These results corroborate the idea that at interplanetary sector boundaries, cometary disconnection events via magnetic reconnection are induced (Niedner \& Brandt 1978; Yi et al. 1996; Brandt et al. 1999).

Note, however, that even in the case of a homogeneous solar wind, disconnection events in the inner tail can, in principle, occur, though they are expected to be less pronounced than the ones initiated by dayside reconnection. They are triggered by pinching of the outer elongated magnetic barrier that partly enwraps the cometotail and by dayside diffusion.

A localized violation of the ideal Ohm's law, i.e. anomalous resistivity, is essential for the reconnection scenarios we described in this contribution. The inhomogeneities in density and temperature of plasma at the cometary boundaries provide a free energy source that inescapably leads to the excitation of drift instabilities that can be modeled by means of a currentdependent resistivity. The actual microphysics of the anomalous transport is rather involved. In this respect, our present studies are a first step towards a better understanding of the complex interaction of the hot solar wind plasma and the cold cometary gas.

Acknowledgements. We thank J.C. Brandt for his valuable examination of the original version of our manuscript.This work was supported by the Deutsche Forschungsgemeinschaft, DFG project LE 1039/5-1,4-2.

\section{References}

Alfvén, H. 1957, Tellus, 9, 92

Barnard, E. E. 1899, MNRAS, 59, 354

Benz, A. O. 1993, Plasma Astrophysics (Dordrecht: Kluwer)

Birk, G. T., \& Otto, A. 1996, J. Comp. Phys., 125, 513

Brandt, J. C., \& Mendis, D. A. 1979, in Solar System Plasma Physics, ed. C. F. Kennel, L. J. Lanzerotti, \& E. N. Parker (Amsterdam: North-Holland), p. 255 
Brandt, J. C., \& Niedner Jr., M. B. 1987, A\&A, 187, 281

Brandt, J. C., Caputo, F. M., Hoeksema, J. T., et al. 1999, Icarus, 137, 69

Brandt, J. C., \& Snow, M. 2000, Icarus, 148, 52

Brosius, J. W., Holman, G. D., Niedner Jr., M. B., et al. 1987, A\&A, 187,267

Cremonese, G., \& Fulle, M. 1988, A\&A, 202, L13

Flammer, K. R., Mendis, D. A., \& Shapiro, V. D. 1997, ApJ, 482, 1021

Gombosi, T. I., De Zeeuw, D. L., Häberli, M., \& Poweel, K. G. 1996, J. Geophys. Res., 101, 15233

Huba, J. D. 1985, in Unstable Current Systems and Plasma Instabilities in Astrophysics, IAU 107, ed. M. R. Kundu, \& G. D. Holman (Dordrecht: Reidel), p. 315

Huba, J. D. 1998, NRL Plasma Formulary, Revised Edition 1998, Naval Research Laboratory, Washington DC

Ip, W.-H. 1985, ESA SP-235, Proc. ESA Workshop on Future Mission in Solar, Heliospheric, and Space Plasma Physics, p. 65

Kirsch, E., McKenna-Lawlor, Daly, P., et al. 1989, Ann. Geophys., 7, 107

Kivelson, M. G., \& Russell, C. T. 1995, Introduction to Space Physics (Cambridge: Cambridge University Press)

Konz, C., Birk, G. T., \& Lesch, H. 2004, A\&A, submitted
Mendis, D. A., \& Ip, W.-H. 1977, Space Sci. Rev., 20, 145

Niedner Jr., M. B., \& Brandt, J. C. 1978, ApJ, 223, 655

Niedner Jr., M. B., Ionson, J. A., \& Brandt, J. C. 1981, ApJ, 245, 1159

Niedner Jr., M., \& Schwingenschuh, K. 1987, A\&A, 187, 103

Ogino, T., Walker, R. J., \& Ashour-Abdalla, M. 1986, Geophys. Res. Lett., 13(9), 929

Papadopoulos, K. 1977, Rev. Geophys. Sp. Sci., 15, 113

Rauer, H., Wegmann, R., Schmidt, H. U., \& Jockers, K. 1995, A\&A, 295, 529

Russel, C. T., Saunders, M. A., Phillips, J. L., \& Fedder, J. A. 1986, J. Geophys. Res., 91, 1417

Schmidt-Voigt, M. 1989, A\&A, 210, 433

Smith, E. J., Balogh, A., Forsyth, R. J., \& McComas, D. J. 2001, Geophys. Res. Lett., 28, 4159

Verigin, M. I., Axford, W. I., Gringaux, K. I., \& Richter, A. K. 1987, Geophys. Res. Lett., 14, 987

Voelzke, M. R., \& Matsuura, O. T. 2000, A\&AS, 146, 1

Wegmann, R. 2000, A\&A, 358, 759

Yi, Y., Caputo, F. M., \& Brandt, J. C. 1994, Planet. Space Sci., 42, 705

Yi, Y., Walker, R. J., Oginao, T., \& Brandt, J. C. 1996, J. Geophys. Res., 101, 27585 